\title{
EFEITO POTENCIALIZADOR DO Cymbopogon citratus-Stapf SOBRE A AÇĀO DA ACETILCOLINA NA CONTRATILIDADE DE MŨSCULO LISO
}

Ana Maria Külzer

Curso de Fisioterapia. Centro de Ciēncias da Saūde. UFSM. Santa Ma ria, RS.

Amauri Rodrigues da Silva e Zuleica Tabarelli.

Departamento de Fisiologia. Centro de Ciências da Saúde. UFSM. Santa Maria, RS.

RESUMO

Os autores pesquisaram a ação do extrato aquoso à $2 \%$ de Cymbopogon citratus-stapf sobre a contratilidade de músculo liso (du odeno) de rato wistar, machos. Foram realizados 10 experimentos; ca da um constou de registro de contratilidade de um fragmento de duo deno de rato Wistar, mantido em banho-maria para órgão. isolado, com solução de Tyrode, controlando-se os padrões fisiológicos de tempe ratura, pH e oxigenação do banho, a fim de garantir a natureza fun cional da contratilidade do mesmo.

Para cada experimento realizavam-se três registros, na se guinte sequência:

1. Registro padrão (controle) de contratilidade do órgão no banho (Tyrode);

2. Registro teste: após se acrescentar ao banho acetilco lina. Decorridos 15 minutos de substituição do banho e lavagem do ōrgão;

3. Registro experimental: após colocação do chá mais ace tilcolina no banho.

Verificou-se que houve diferença significativa entre os grupos 2 e 3 em relação ao grupo controle na motricidade de músculo liso de duodeno isolado, concluindo-se assim que o Cymbopogon citra tus produziu efeito acetilcolina similis.

SUMMARY

KOLZER, A.M.; SILVA, A.R. da and TABARELLI, Z., 1990. Potentializing effect of Cymbopogon citratus - Stapf on acetylcholine action in smooth muscle contration. Ciência e Natura, 11: $83-92,1989$

Authors searched actions of Cymbopogon citratus-Stapf aquous extract, $2 \%$ concentration, on montility of smooth muscle.

It were performed 10 (ten) experiments, each of them con sisted of the motility record of a segment of duodenum from male wistar rats maintained in a Tyrode solution water-bath to isolated organ. Physiological parameters (temperature, pH, oxigen supply) were 
controled to obtain the functional nature of organ motility.

It were realized three records to each experiment in this sequency:

First record: control record (standard) from motility of duodenum smooth muscle in Tyrode bath.

Second record: after addition of $0,5 \mathrm{ml}$ physiological so lution before the third record, the bath liquid was entirely substi tuted and the organ was washed tith Tyrode solution.

Third record: after 15 (fifteen) minutes from the addition of Cymbopogon citratus-Stapf tea $2 \%$ concentration, it was add $0,5 \mathrm{ml}$ acetylcholine solution (11,8165 Mg in $50 \mathrm{ml}$ physiological solution).

It was verified that it shows a significant difference between 2nd and third record groups compared whit control records (first record groups) on smooth muscle motility of isolated duodenum from wistar male rats.

INTRODUÇAOO

0 gēnero Cymbopogon citratus-stapf è uma planta da familia das gramīneas, chamada vulgarmente de capim-cidrão, capim-limão e impropriamente chamado em algumas regiões de erva-cidreira. E encon trada às margens das estradas do sul do Brasil (15). Trata-se de planta perene, denso-creptosa, adventícia, de touceiras grandes, que atingem em média $100 \mathrm{~cm}$ de altura; possui rizomas curtos, colmosere tos e glabros, margens escrabosas e, no āpice, medindo aproximada mente $100 \times 5-15 \mathrm{~mm}$ inflorescēncia de $30-50 \mathrm{~cm}$. Figuras 1,2 e 3 .

Não suporta grandes altitudes e temperaturas muito baixas. E usada para chās estomacais (2). 0 chā de suas folhas tem proprie dades calmantes (12). Triturada exala aroma de limão. Origināria da India, introduzida no Brasil nos tempos coloniais, e cultivada camo ornamental e medicinal.

os autores (30), referem-se a outros nomes vulgares: ca pim-santo, citronela, capim-cheiroso.

E cultivada em hortas, jardins e plantado à beira de estra da em Santa Catarina como cerca viva.

0 Cymbopogon citratus-Stapf é utilizado na Medicina popu lar como calmante, analgēsico em dores de estōmago, abdominais e de cabeça; antifebril, anti-reumātico, carminativo, antitussīgeno, dia forético, enemagogo e em distūrbios digestivos. Também tem uso do méstico popular como repelente de insetos.

Utilização farmacêutica: utilizado industrialmente como fonte de citral, que é empregada com flavorizante na indústria de alimentos e cosméticos, alēm de constituir matēria-prima na sỉntese de iononas e vitamina $A(30)$. 


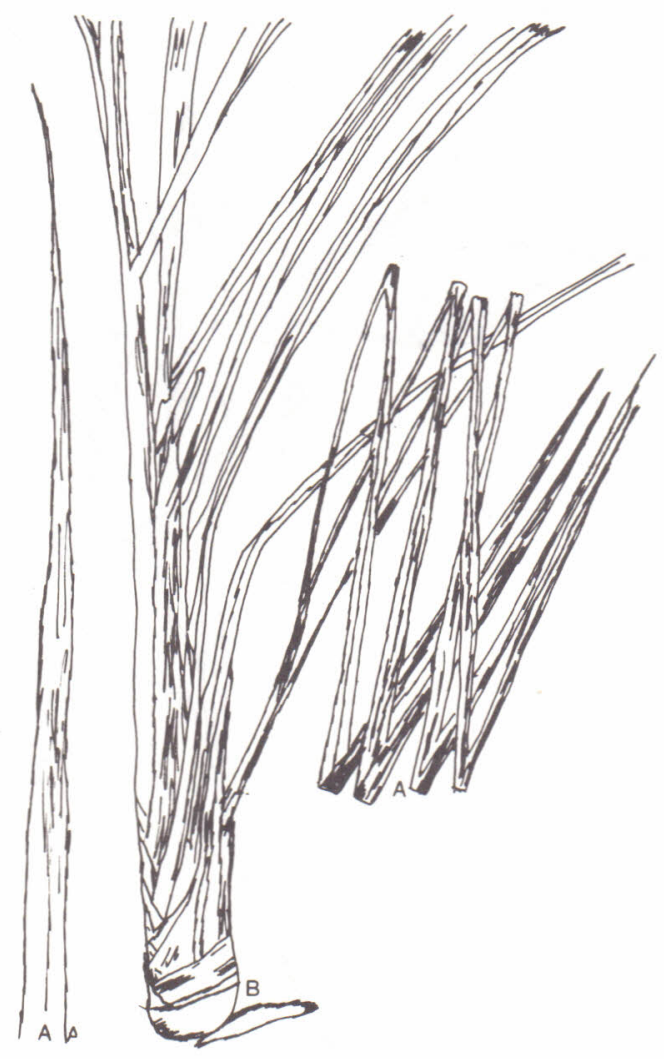

Figura 1 - Cymbopogon citratus (DC) Stapf. A - Aspecto da folha. BBase da planta com as bainhas.

Dados químicos e farmacológicos: as folhas possuem óleo essencial ( 3 e 4 ) uma saponina (provavelmente o glucosideo do fucos terol e sitosterol, dois álcoois saturados de cadeia longa e um al calóide não identificado (21). Nos rizomas e nas folhas foi detecta da a presença de alcalóides indólicos (5).

Da cera que recobre o capim-cidró foram isolados 2 tripte nóides, uma cetona (Cymbopogona) e um àlcool (Cympogonol) (6 e 14).

o óleo essencial é constituido de ácidos voláteis, alco ois, aldeỉdos, cetonas, ésteres, terpenos, sesquiterpenos (4, 17 e 34) predominando o citral, o mirceno e geraniol. Dependendo da regiãoda coleta o material apresenta variaçōes considerāveis, tanto do ponto de vista qualitativo como quantitativo $(1,4,20,22,25,27,29$ e 33).

0 óleo essencial mostrou atividade antibacteriana e fung $\underline{i}$ cida (7, 16 e 26). Esta propriedade é atribuida ao citral, sendo que 
- mirceno não apresenta, mas possui a capacidade de aumentar a at $\underline{j}$ vidade antibacteriana do citral (23).

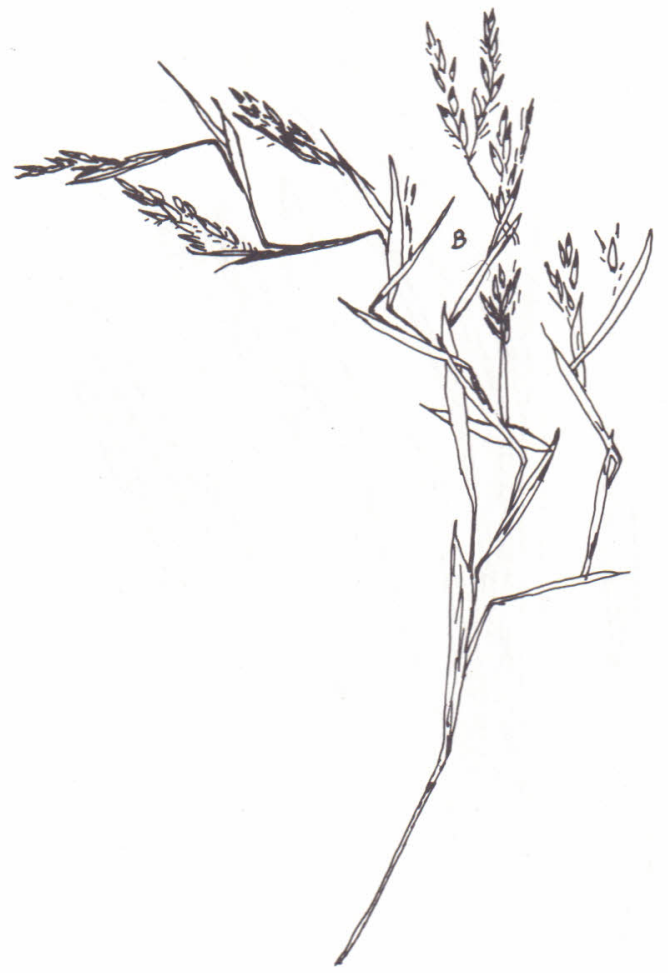

Figura 2 - Cymbopogon citratus (DC.) Stapf. Inflorescência.

o hidrolato, o pseudo-hidrolato das folhas do capim-cidró e o citral isolado, mostram atividades anti-espasmödicas e relaxan te da musculatura lisa de animais de experimentos (11).

Atividades analgésicas, anti-pirētica, anti-oxidante e de pressora do SNC são tambēm relatados (13 e 28).

Foram estudados no programa de Pesquisa de Plantas Medici nais, apoiada pela CEME (Central de Medicamentos) do Ministērio de Previdência e Assistência Social, a ação de chä e infusões, quanto às suas propriedades calmantes. A ação sedativa não foi comprovada nem nos modelos experimentais usados, nem nos ensaios clínicos (30). Possui poder inseticida (18 e 32 ).

o Cymbopogon citratus-stapf ordinariamente não floresce no estado do RS, e/ sō aparece se cultivado. Pode ser plantado ao f $\underline{i}$ nal do inverno e na primavera (2). Seu óleo essencial extraỉdo das folhas, tem odor de cedro sendo empregado na perfumaria. E cultiva da tambēm na Argentina (8). 


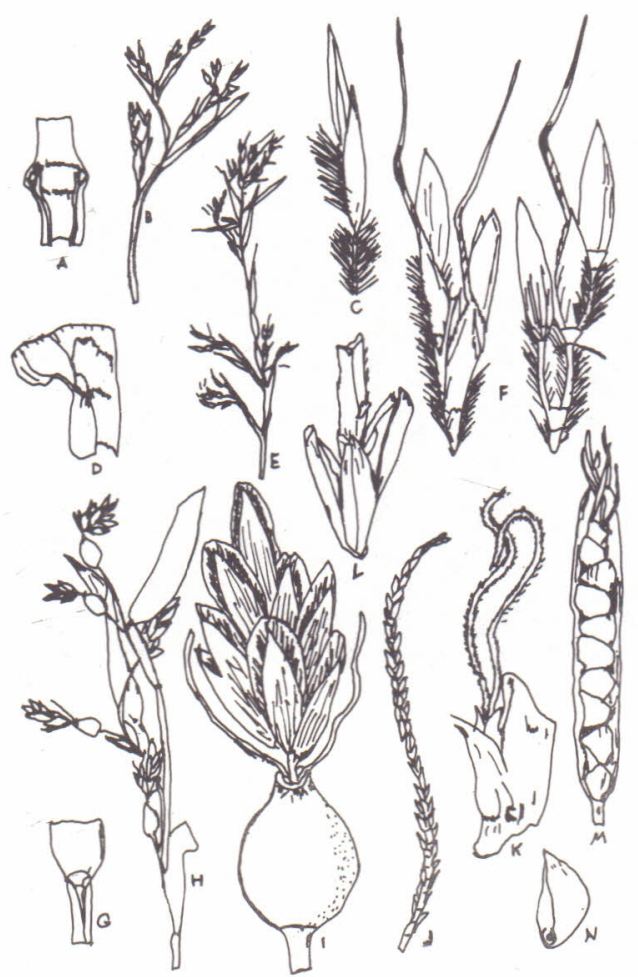

Figura 3 - Cymbopogon citratus (DC.) Stapf: segundo Araújo(2).

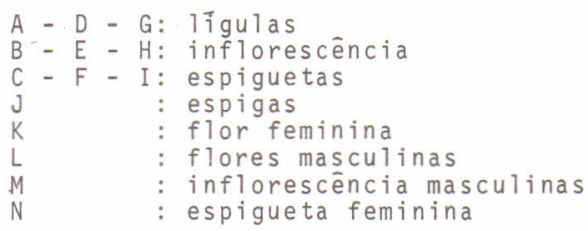

Segundo Pio Correia (4), das plantas extrai-se, em numero sos países, 3 a $4 \%$ do óleo essencial volátil. Usado largamente na economia doméstica de vārios povos, como excitante difuso, anti-reu mático, no tratamento de paralisias e de úlceras atōnicas. Em algu mas colonias portuguesas serve para aromatizar a aguardente, e os hindus usaram-na para imprimir em certos tecidos um aroma que os dis tinguia de outros tecidos congêneres de outras procedências.

E usado como planta forrageira e para cobertura de cabanas (31). Devido ao seu uso popular, os autores pesquisaram os efeitos do chā de Cymbopogon citratus à $2 \%$ na contratilidade de músculo li so de duodeno isolado quando usado em banho nutriente simultaneamen te com acetilcolina. Figura 4. 


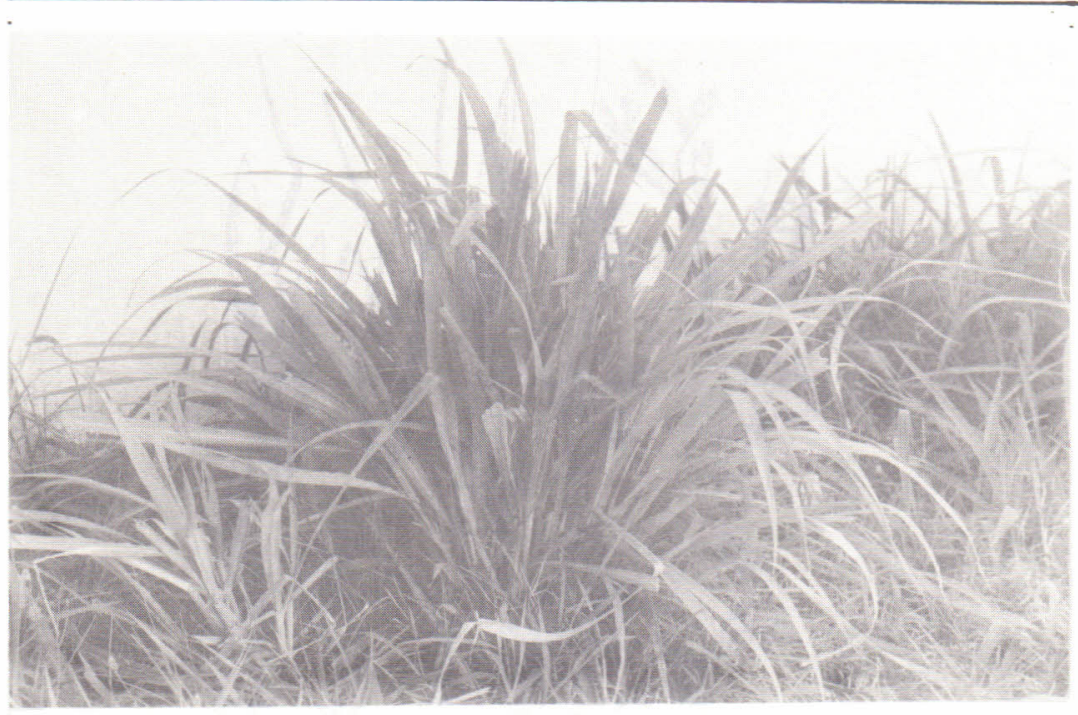

Figura 4 - 0 Cymbopogon citratus - Stapf: aspecto da planta.

MATERIAL E METODOS

Foram utilizados, para a obtenção dos eletromiogramas de músculo liso, 10 segmentos de duodeno de ratos wistar, machos. Os animais foram sacrificados por pancada na nuca, e, rapidamente, ti nham o abdomem aberto e retirado um segmento de aproximadamente 04 $\mathrm{cm}$, da primeira porção do duodeno. O segmento de intestino era colo cado numa placa de Petry com solução de Tyrode, aquecida $\bar{a} 37^{\circ} \mathrm{C}$.

Usando-se uma seringa hipodērmica, era lavado o segmento do intestino no sentido craneo-cauda, com a mesma solução, à mesma temperatura. Após este procedimento, o intestino era transferido pa ra um banho-maria de ōrgão isolado, à fim de se proceder os regis tros. o banho é constituīdo de solução de Tyrode (24).

os eletromiogramas foram realizados com o transdutor de força acoplado ao eletromiograma do aparelho registrados marca F siōgrafo (Texas Instruments-Houston - Texas).

Para cada segmento de intestino foram feitos três regis tros:

- Registro padrão (controle).

- Registro teste: com solução de acetilcolina para compro var a resposta contrátil normal do músculo.

Procedeu-se apōs isso lavagem do ōrgão com substituição do banho e,

- Registro experimenta 1: com solução de chā, sendo a ace tilcolina (Ach) acrescentada apōs 15 minutos da incubação do chà. 
Os registros foram analisados medindo-se a amplitude das contrações (em $\mathrm{cm}$ ).

Os dados foram tabelados analisados pelo Teste " $t^{n}$ de Stu dent pareado, pela anālise de variāncia e pelo Teste de Tuckey-(9). RESULTADOS

Apresentados na Figura 5 e na Tabela I:

A anālise dos resultados mostrou que os tratamentos acetilcolina (tes te) e chá mais acetilcolina (experimental) diferem significativamente do registro padrão - nível de significância 0,5, conforme pode ser observado na Tabela I.

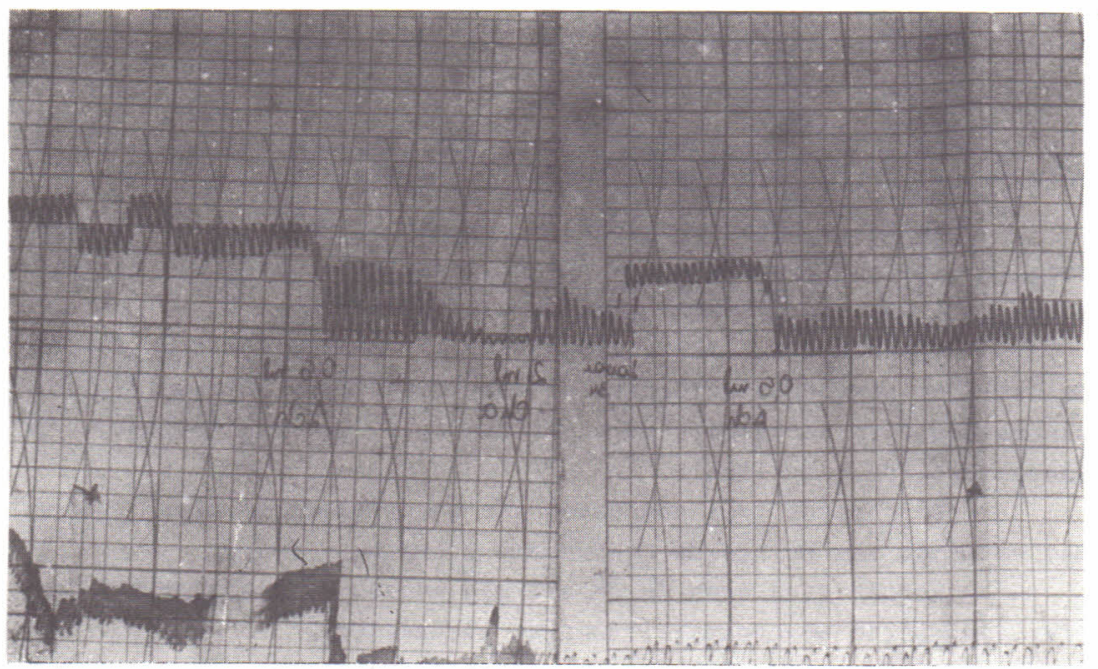

Figura 5 - Registro grāfico da ação do Cymbopogon citratus Stapf.

- A: registro basal, seguindo-se a ação da acetilcolina(Ach), extrato aquoso de Cymbopogon citratus Stapf e acetilcoli na.

\section{DISCUSSÃO}

Várias plantas da flora brasileira possuem efeito sobre a contratilidade do músculo liso. E o caso da Metissa officinalis, L (melissa), do cechorim intybus, L (chicōria-amarga) (19), de Atropa beladona (beladona), da Matricaria chamomila (camomila), da Pimpine Ia anisium (erva-doce), etc. (3 e 19).

Essas plantas são usadas pelo povo nos distūrbios da at 1 vidade digestiva, como cōlicas, diarréias, nos espasmos uterinos du rante o parte e nos vômitos.

o Cymbopogon citratus, tambēm é usado popularmente como anti-espasmōdico para alivio das cōlicas, isto é, como relaxante da musculatura lisa do intestino. 
TABELA I - VALORES MEDIOS EXPRESSOS EM cm, DA AMPLITUdE DE CONTRA ÇOES DE MUSCULO LISO DE DUODENO ISOLADO DE RATOS WISTAR MACHOS NA VIGENCIA DE ACETILCOLINA MAIS EXTRATO AQUOSO BRUTO A $2 \%$ DE Cymbopogon citratus - Stapf.

\begin{tabular}{ll}
\hline \hline Tratamento no Banho & $\begin{array}{l}\text { Valores Médios (em } \mathrm{cm} \text { ) da } \\
\text { amplitude de contrações. }\end{array}$ \\
\hline $\begin{array}{l}\text { Grupo Testemunha } \\
\text { Acetilcolina }\end{array}$ & 0,61 \\
$\begin{array}{l}\text { Acetilcolina mais } \\
2 \text { ml Extrato Aquoso }\end{array}$ & $3,35^{*}$ \\
de Cymbopogon citra- & \\
tus à 2\%
\end{tabular}

* Difere significativamente do grupo testemunha $P \leq 0.05$.

Muito se pesquisou sobre o Cymbopogon citratus-stapf, mas pouco se sabe sobre o efeito na musculatura lisa, sendo que no caso de cōlica é onde se encontra o maior uso popular.

Ferreira, em 1983 e 1984 (10, 11), provou que trēs compos tos isolados possuem atividades anti-espasmödicas e relaxantes da musculatura lisa de animais de experimentação. São o hidrolado, o pseudohidrolado e o citral.

Ora, esses trēs compostos são encontrados no ōleo essen cial extraido das folhas de Cymbopogon citratus - Stapf.

Portanto, a ação de um extrato aquoso da planta deveria pro vocar diminuição da contratilidade da musculatura.

os nossos resultados, no entanto, mostraram que o chā tem ação semelhante à da acetilcolina, isto é, aumentava a amplitude da contração do músculo liso.

A amplitude da contratilidade nos experimentos mostram que, no registro basal, a média foi $0,61 \mathrm{~cm}$; usando-se acetilcolina, a média se eleva para $2,64 \mathrm{~cm}$. Apōs lavagem do örgão e substituição do banho, acrescentando-se $2 \mathrm{ml}$ de chā a. $2 \%$ e após 15 minutos acres centando-se acetilcolina; esperava-se que, como depressor da ativi dade da musculatura lisa, a mëdia deveria ser menor. No entanto, ela cresceu para $3,35 \mathrm{~cm}$.

Acreditamos que, se os pacientes acusam melhora dos sin tomas produzidos por aumento da contratilidade do músculo liso com a administração do chā de Cymbopogon citratus, este fato se deva a uma estimulação simpática, produzida pelo excesso de motilidade pro vocada pela ação parassimpática. Talvez o chā estimule o SN Paras simpātico e, como efeito corretor, os centros simpáticos atuem de pois, melhorando os sintomas apresentados pela hipermotilidade. 
CONCLUSÃO

Dos resultados obtidos, podemos concluir:

- o extrato aquoso de Cymbopogon citratus (Stapf) potencializa os efeitos da acetilcolina no músculo liso de duodeno isolado.

- Se o povo o usa como anti-espasmódico, hā que existir um mecanis mo corretor endógeno que seja provocado pelo uso do chá da planta.

BIBLIOGRAFIA CONSULTADA

1. ABEgAS, B. \& YohanNes, P.G.J. Nat. Prod. 46(3):424-6, 1983.

2. ARAJjo, A.A. Principais gramineas do Rio Grande do Sul, Porto Alegre, Sulina, 255p.

3. CARVAlho, A.R.D. Acura pelas plantas e diversos meios de grande poder curativo, São Paulo, Folco Masucci, 1970.

4. CORREIA, A.A. et alii: Garcia de Orta, 8(3):629-38, 1960.

5. CORREIA, A.A. et alii: Garcia de Orta, 10(1):103-9, 1962 .

6. CRAWFORD, M. et alii: Tetrahedron Lett, 35:3099-102, 1975 .

7. CHIORI, C.0. et alii: J. Pharmac. Med. Sci., 1:267-70, 1977.

8. DIMITRIM, J. Enciclopēdia Argentina de agricultura e jardinaria. $2^{2}$ a ed. Buenos Aires, Acme S.A.C.I., 1972.

9. DIXON, W.J. \& MASSEY, Jr. F.J. Introduction to Statistical Ana lysis MC Graw Hil1, 3 ed. U.S.A.; 1969, 629p.

10. FERREIRA, M.S.C. et alii: Reunião Anual SBPC, 35 - Belēm, 6-13, julho, 1983.

11. FERREIRA, M.S.C.; \& FONTELES, M.C. Simpōsio de plantas medici nais do Brasi1, 8 - Manaus, 6 set. Resumos, InTA, 1984, 729p.

12. GemTChuJNicov, I.D. Manual de Taxonomia Vegetal: plantas de in teresse econômico. São Paulo, Agronômica ceres, 1976, 368p.

13. GYANE, D.0. Drug. Cosmt. Ind. 118: 36-8-40, 1976.

14. HAUSON, S.W. et alii: Phytochem,15: 1074-5, 1976.

15. 20LY, A.B. Botānica: Introdução e Taxonomia Vegetal, São Paulo, 1975 .

16. KOKATE, C.K. \& VARMA, K.C. Sci. Cult. 37:196, 1971.

17. LEUNG, A.Y. Encyclopedia of common natural ingrediente used in food, drugs and cosmetics. New York, 1980.

18. MARTINDALE: The Extra Pharmacopeia, 280 ed., London Pharmaceut cal, 2025, 1982 .

19. MOREIRA, F. As plantas curam, São Paulo, Hemus, 1971.

20. NEYBERG, A.G. Bull Agr. Congo Belga, 44:319, 1953.

21. OLANIYI, A.A. et alii: Planta Médica. 28, 186-9, 1975.

22. OLIVEROS, B.L. \& AUREUS, E. Int. Cong. Essent. 0i1s. 7:166, 1979.

23. ONAWUNMI, G.0. et alii: J. Ethno pharmacol. 12(3):279-86, 1984.

24. PERRY, W.L.M. Pharmacological experimentoon isolected predara tions, S. Livingston, London, $163 p$.

25. RABHA, L.C. et alii: Indian perfum. 23:178, 1979. 
26. RAMADAN, F.M. et alii: Chem. Mikribiol. Technol., Lebensm, 1: $96,1972$.

27. ROVESTI, P. \& VARIATI, G.L. Fr. Ses. Perfums, 3:39, 1960.

28. SETH, G. et alii: Indian, J. Exp. Bid., 14:370, 1976.

29. SILVA, G.A.A.B. \& BAUER, L. Rev. Bras. Farma., 52(4):193-6, 1971.

30. SIMOES, C.M.0.; MENTZ, L.A. Schenkel, e.p., Irgang, B.E. e Steh mann, J.R. Plantas da medicina popular do Rio Grande do Sul, ed. da UFRGS, Porto Alegre, 1986.

31. SMITH, L.B. Wasshansen, DC Klain, P.M. Flora Ilustrada Catari nense-Gramineas, Raulino Reitz, Itajaí, 1982, 174 p.

32. SOFOWORA, E.A. \& ISAAVES, W.A. Nat. Prod., 34: 383, 1971.

33. SYLVA, M.G. de Mfq. Chemist., 30:415, 1959.

34. VALE, J.C. Bol. EsC. Farm. 629-38, Portuga1, 1966.

Recebido em dezembro, 1989; aceito em fevereiro, 1990. 